\title{
Using Interocular Suppression and EEG to Study Semantic Processing
}

\author{
Tom Heyman and Pieter Moors \\ Department of Psychology, University of Leuven, 3000 Leuven, Belgium \\ Review of Kang et al.
}

Presenting an image to one eye and a dissimilar image to the other eye induces a phenomenon called binocular rivalry, in which the conscious percept alternates between the two dissimilar images despite constant retinal input. This technique has provided some insight regarding the levels of visual processing that can take place outside awareness (for review, see Lin and $\mathrm{He}, 2009)$. To meticulously study the neural correlates of awareness, binocular rivalry has, however, some disadvantages. Perceptual dominance durations only last a few seconds and switches are highly unpredictable. To ensure a long-lasting stable percept, Tsuchiya and Koch (2005) introduced a technique called continuous flash suppression (CFS), in which one of the eyes is presented with a dynamic noise pattern changing typically every $100 \mathrm{~ms}$. Such a mask has proven to effectively dominate over the image presented to the other eye, rendering it consciously invisible.

It remains unclear whether flashsuppressed stimuli are processed up to the semantic level. Originally, Zimba and Blake (1983) reported that no semantic priming occurred when prime words were suppressed from awareness through bin-

\footnotetext{
Received Nov. 15, 2011; revised Dec. 11, 2011; accepted Dec. 13, 2011. We thank Lee de-Wit and Jonas Kubilius for their comments and help with writing the paper. We also thank Gert Storms, Loes Stukken, and Raymond van Ee for clarifying comments on earlier versions.

The authors declare no competing financial interests.

Correspondence should be addressed to Tom Heyman, Department of

Psychology, University of Leuven, Tiensestraat 102, 3000 Leuven, Belgium.

E-mail: tom_heyman123@hotmail.com.

DOI:10.1523/JNEUROSCI.5726-11.2012

Copyright $\odot 2012$ the authors $\quad 0270-6474 / 12 / 321515-02 \$ 15.00 / 0$
}

ocular rivalry. However, a recent study showed that participants see a target word faster through a CFS mask (i.e., break through suppression) when it was preceded by a related visible prime word (Costello et al., 2009). In a recent issue of The Journal of Neuroscience, Kang et al. (2011) tried to resolve this debate by combining interocular suppression and EEG recordings. Specifically, they focused on N400, a negative peak occurring $\sim 400 \mathrm{~ms}$ after an unexpected stimulus, which is associated with semantic processing (Kutas and Federmeier, 2011). Previous studies demonstrated that words elicit smaller N400 amplitudes when preceded by semantically related primes than when preceded by unrelated primes (for references, see Kang et al., 2011). Building on these results, Kang et al. (2011) used interocular suppression to render either prime or target words invisible and investigate how this affects N400.

In Experiments 1, 2, and 4, Kang et al. (2011) used CFS to render target words invisible after a prime word was presented. Participants had to signal whether the target word was related or unrelated to the prime word. Participants did not perform better than chance on suppression trials and did not show an N400 effect. Thus, there was neither a behavioral nor a neural priming effect. In contrast, on control trials (i.e., trials with clearly visible targets) performance was high and an N400 effect was observed. These results were interpreted to mean that suppressed words are not semantically processed.
To properly interpret the results of Kang et al. (2011), however, it is important to consider three points regarding N400. Although Kang et al. (2011) found an N400 effect on control trials, there was no N400 component observed. Whereas the former refers to a difference in negativity $\sim 400 \mathrm{~ms}$ between two conditions (Kang et al., 2011, their Fig. 2 B), the latter simply indicates the occurrence of a negative peak $\sim 400 \mathrm{~ms}$ after presentation of an unexpected stimulus (Kang et al., their Fig. 3B, red line; Kutas and Federmeier, 2011). Given that both primes and targets were clearly visible in the control conditions, a typical negative peak at $\sim 400 \mathrm{~ms}$ would be expected, but none was present.

Regarding the lack of an N400 effect in the experimental conditions used by Kang et al. (2011), it is possible that a difference in negativity occurred later than $400 \mathrm{~ms}$ after presentation of the target word. Most priming studies investigating the influence of awareness use subliminally presented primes and supraliminal targets. To our knowledge, only a few studies used an approach like that of Kang et al. (2011), i.e., masked targets in combination with ERP measurements. These studies reveal an N400 effect, but with delayed latency [e.g., $812 \mathrm{~ms}$ (Wang and Yuan, 2008)]. Visual inspection of the ERP waveforms from Kang et al. (2011) does not show evidence of a delayed N400 effect, but their time window was limited to $800 \mathrm{~ms}$, and thus a delayed effect may not have been detected.

Moreover, the nature of N400 is still a point of discussion (Kutas and Feder- 
meier, 2011). Some argue that it is modulated by automatic semantic processes. Others claim that it merely reflects a postlexical process whereby the target word is integrated in the context (for review, see Kutas and Federmeier, 2011). The latter approach assumes that the N400 effect disappears when target words are suppressed from consciousness. Depending on which view one adopts, the results of Kang et al. (2011) can be interpreted differently. If one advocates the automatic process account, the absence of an N400 effect indicates that interocular suppression prevents semantic analysis. Alternatively, these findings could be considered as evidence in favor of the postlexical process account, since no N400 effect was found when participants were unaware of the target words. According to this explanation, interocular suppression impedes postlexical processes; no such claim is made about automatic lexical access. In other words, some semantic processes may occur in the absence of awareness induced by interocular suppression.

On a methodological note, it should be stressed that participants whose semantic judgment accuracy under suppression was $>60 \%$ were removed from the data. The rationale for this exclusion was that highly accurate participants probably experienced incomplete suppression. Such suppression problems may seem surprising, given that CFS is often described as a readymade tool for effectively suppressing any stimulus, but in fact a CFS mask can fail if not properly tuned to the suppressed stimulus. Nevertheless, the finding that performance for suppression trials was at chance is not surprising given the $60 \%$ criterion. It may have been preferable to include participants with high semantic judgment accuracy, because nothing in the setup of Experiments 1 and 2 excludes the possibility that participants with a score $>60 \%$ were actually processing target words subliminally. An N400 effect in this group of participants might be the consequence of an ineffective mask and thus consciously processing the target word. Alternatively, it might be the result of unconsciously processing the target word, which in turn affected both performance and ERP signals. Instead of discarding these subjects, the alternative explanations for their high scores could be dissociated by using a response option for stimuli that broke through suppression, a method which has been used before (Yang et al., 2010).

In Experiment 3 by Kang et al. (2011), binocular rivalry was used to suppress primes instead of targets. Here, participants had the opportunity to indicate when a prime word was recognized during suppression (so-called discard trials). Two points should be noted when looking at the results. First, an N400 component seems to be present in both dominance and suppression trials. Second, a significant N400 effect was observed in dominance trials. Surprisingly, an N400 effect was also observed in suppression trials for the high-discard group (average discarded rate of $18 \%$ per participant) and not in the low-discard group (average discard rate of 7\%). The authors attributed the N400 effect to partial visibility of the primes; this conclusion was supported convincingly by Experiment 4, in which target visibility was manipulated. Still, we believe that attention might also play an important role, since it has been shown to modulate N400 priming effects (McCarthy and Nobre, 1993). Furthermore, Bahrami et al. (2008) showed that spatial attention can guide orientation processing of Gabor patches rendered invisible through CFS. Therefore, differences in attention might explain why only some participants showed an N400 effect (i.e., those who were highly attentive). Attention might have played a role in the other experiments as well. Since participants had to indicate whether two words were related or unrelated, they may have developed a strategy of guessing in certain stimulus contexts because they were only aware of the meaning of one word, as the other word was suppressed from consciousness. When participants learn to discriminate early between control (normal) and suppression (foils) trials, they might shift their attention away in these latter trials. As a consequence, the N400 priming effect might be attenuated in the suppression condition.
In summary, the study of Kang et al. (2011) used interocular suppression in combination with EEG in an attempt to resolve the issue regarding semantic processing of suppressed stimuli. Throughout four experiments, no N400 effect was found when words were reliably suppressed from awareness. This led the authors to conclude that suppression thwarts semantic analysis. However, there is no consensus in the literature regarding the nature of the N400 effect. Consequently, the results can be interpreted as the result of an automatic or a postlexical process. Furthermore, we raise the possibility that the N400 effect might in fact be delayed rather than missing and point to the unexpected absence of an N400 component in control trials. Despite possible theoretical and methodological issues, this paradigm provides a good starting point for future research.

\section{References}

Bahrami B, Carmel D, Walsh V, Rees G, Lavie N (2008) Spatial attention can modulate unconscious orientation processing. Perception 37:1520-1528.

Costello P, Jiang Y, Baartman B, McGlennen K, He S (2009) Semantic and subword priming during binocular suppression. Conscious Cogn 18: 375-382.

Kang MS, Blake R, Woodman GF (2011) Semantic analysis does not occur in the absence of awareness induced by interocular suppression. J Neurosci 31:13535-13545.

Kutas M, Federmeier KD (2011) Thirty years and counting: finding meaning in the N400 component of the event-related brain potential (ERP). Annu Rev Psychol 62:621-647.

Lin Z, He S (2009) Seeing the invisible: the scope and limits of unconscious processing in binocular rivalry. Prog Neurobiol 87:195-211.

McCarthy G, Nobre AC (1993) Modulation of semantic processing by spatial selective attention. Electroencephalogr Clin Neurophysiol $88: 210-219$.

Tsuchiya N, Koch C (2005) Continuous flash suppression reduces negative afterimages. Nat Neurosci 8:1096-1101.

Wang Q, Yuan J (2008) N400 lexicality effect in highly blurred Chinese words: evidence for automatic processing. Neuroreport 19:173-178.

Yang E, Hong SW, Blake R (2010) Adaptation aftereffects to facial expressions suppressed from visual awareness. J Vis 10:24.

Zimba LD, Blake R (1983) Binocular rivalry and semantic processing: out of sight, out of mind. J Exp Psychol Hum Percept Perform 9:807815. 